\author{
NASA Contractor Report 191476 \\ NASA-CR-191476 \\ 19940008379
}

ICASE Report No. 93-27

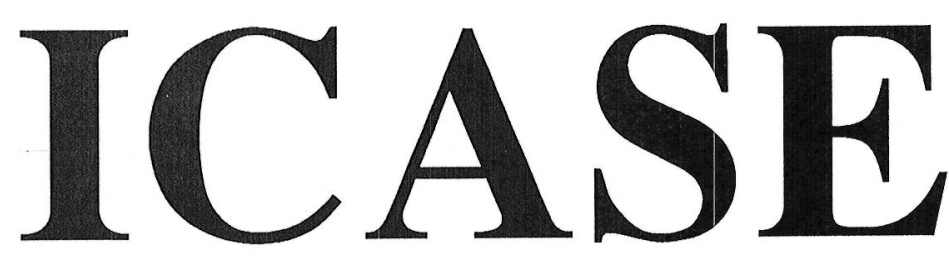

\title{
A COMPARISON OF TWO FORMULATIONS FOR HIGH-ORDER ACCURATE ESSENTIALLY NON-OSCILLATORY SCHEMES
}

\section{Jay Casper \\ Chi-Wang Shu \\ H. L. Atkins}

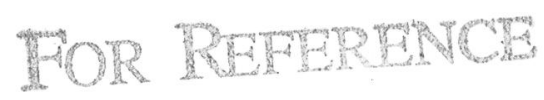

NASA Contract No. NAS1-19480

June 1993

Institute for Computer Applications in Science and Engineering

NASA Langley Research Center

Hampton, Virginia 23681-0001

Operated by the Universities Space Research Association

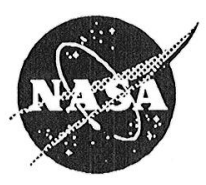

National Aeronautics and Space Administration

Langley Research Center

Hampton, Virginia 23681-0001
LIBRARY COPY 



\title{
A COMPARISON OF TWO FORMULATIONS FOR HIGH-ORDER ACCURATE ESSENTIALLY NON-OSCILLATORY SCHEMES
}

\author{
Jay Casper ${ }^{1}$ \\ ViGYAN, Inc. \\ 30 Research Drive \\ Hampton, VA 23666 \\ Chi-Wang Shu \\ Division of Applied Mathematics \\ Brown University \\ Providence, RI 02912 \\ H. L. Atkins ${ }^{3}$ \\ Computational Aerodynamics Branch \\ NASA Langley Research Center \\ Hampton, VA 23681
}

\begin{abstract}
The finite-volume and finite-difference implementations of high-order accurate essentially non-oscillatory shock-capturing schemes are discussed and compared. Results obtained with fourth-order accurate algorithms based on both formulations are examined for accuracy, sensitivity to grid irregularities, resolution of waves that are oblique to the mesh, and computational efficiency. Some algorithm modifications that may be required for a given application are suggested. Conclusions that pertain to the relative merits of both formulations are drawn, and some circumstances for which each might be useful are noted.
\end{abstract}

\footnotetext{
${ }^{1}$ Research supported by U.S. Government Contract NAS1-19672.

${ }^{2}$ Research supported by the National Aeronautics and Space Administration under NASA Contract No. NAS1-19480 while the author was in residence at the Institute for Computer Applications in Science and Engineering (IC:ASE), NASA Langley Research Center, Hampton, VA 23681-0001. Research also supported by U.S. Government Grant NAG1-1145.

${ }^{3}$ Research Scientist, National Aeronautics and Space Administration.
} 



\section{Introduction}

Two basic formulations, finite-volume and finite-difference, for the implementation of high-order accurate, essentially non-oscillatory (ENO) shock-capturing schemes have been the subject of considerable interest in recent years. These schemes achieve high-order spatial accuracy in smooth regions by means of a piecewise polynomial approximation operator that is also designed to avoid oscillations associated with interpolation across steep gradients. As such, they are well suited for the study of aeroacoustic and transition-related problems and may serve as an alternative to spectral methods for solving such problems when shocks or complex geometries are involved.

The finite-volume implementation, first presented by Harten et al. ${ }^{1}$, is preferred for its strict adherence to the integral form in which conservation laws are defined. The primary motivation for the use of the finite-difference approach of Shu and Osher $^{2}$ is computational efficiency. These formulations are briefly described, after which results of their numerical implementations are presented for comparison. The intent of this work is to acquaint the reader with the relative merits of both formulations, the circumstances for which each might be useful, and some details of implementation that may be required for a given application. The performances of both algorithms are compared for accuracy, sensitivity to grid irregularities, resolution of waves that are oblique to the mesh, and computational efficiency.

\section{Discrete Formulations}

The finite-volume and finite-difference algorithms compared in this paper differ fundamentally in the way a system of equations is solved. In both cases, a weak solution of a system of conservation laws is ultimately obtained. The conservation of some quantity $U$ in a spatial domain $D$ can be written

$$
\frac{\partial}{\partial t} \int_{D} U d V=-\int_{\partial D} \overrightarrow{\mathbf{F}} \cdot \overrightarrow{\mathbf{n}} d S
$$

where $\overrightarrow{\mathbf{F}}$ is the flux, $\partial D$ is the boundary of $D, d V$ is a volume element of $D, d S$ is an element of surface area on $\partial D$, and $\vec{n}$ denotes the outward unit normal to $\partial D$.

In the finite-volume approach, the conservation law itself in Eq. (1) is approximated. The spatial domain is discretized, $D=\left\{D_{i}\right\}$, which results in

$$
\frac{\partial}{\partial t} \bar{U}_{i}=-\frac{1}{V_{i}} \int_{\partial D_{i}} \overrightarrow{\mathbf{F}} \cdot \overrightarrow{\mathbf{n}} d S
$$


where $V_{i}$ is the volume of $D_{i}$ and

$$
\bar{U}_{i}=\frac{1}{V_{i}} \int_{D_{i}} U d V
$$

is the cell average of $U$ in $D_{i}$. Eq. (2) is solved for all $i$, which yields a solution $\left\{\bar{U}_{i}\right\}$ of cell averages.

In the finite-difference approach, a pointwise solution is desired. To this end, time differentiation and spatial integration are interchanged in Eq. (2), the divergence theorem is applied on the right-hand side and, in the limit as $V_{i} \rightarrow 0$,

$$
\frac{\partial}{\partial t} U_{i}=-(\nabla \cdot \overrightarrow{\mathbf{F}})_{i}
$$

Special care must be taken when this formulation is implemented, because flux conservation is not as readily achieved as in Eq. (2).

For integration in time, the method of lines will be employed for Eqs. (2) and (4). High-order accurate Runge-Kutta methods, developed by Shu and $\mathrm{Osher}^{2}$, are implemented in the finite-volume and finite-difference schemes to be compared in this paper. Hence, the brief description of the schemes to follow will concern only the right-hand sides of Eqs. (2) and (4).

Both discrete algorithms involve a reconstruction step followed by an evolution step. What is meant by reconstruction is a high-order accurate polynomial approximation at some point in time. In the finite-volume formulation, the solution $U$ is reconstructed from the cell averages to high order within each cell $D_{i}$ and evaluated on the boundary $\partial D_{i}$. The evolution step involves the solutions of the local Riemann problems that arise from the piecewise continuous reconstruction (See, e.g., Refs. 1 and 3 and the references therein.). The spatial integration along the boundary $\partial D_{i}$ is achieved by a correspondingly high-order quadrature. This method will be referred to throughout the paper as ENO-FV. Shu and Osher ${ }^{2,4}$ have proposed the use of the finite-difference formulation in Eq. (4), in which the reconstruction operator is applied directly to the pointwise flux values. The evolution step arises from a fluxvector splitting strategy that is built into the reconstruction. The term ENO-FD will be used to refer to this finite-difference scheme. The pointwise nature of this formulation eliminates the need for dealing with cell averages or the integration of a flux over the boundary of a cell. These distinctions between the two formulations become most important with regard to the issue of cost, and will be discussed in more detail in a later section. 
The most unique aspect of ENO reconstruction operators is their use of adaptive stenciling. At the $i$-th discrete location, the reconstruction set that determines a polynomial $\mathcal{P}_{i}$ is chosen by a searching algorithm in which decisions are based on local smoothness criteria. For example, in one dimension, where the $\left\{D_{i}\right\}$ are intervals on the Real line, Harten et al. ${ }^{1}$ have suggested a hierarchical algorithm that is based on local sets of $k$-th divided differences $\left\{\delta_{j}^{k}\right\}$, where $i-m \leq j \leq i+m$, $k=1,2, \ldots, m$, and $m$ is the desired degree of $\mathcal{P}_{\boldsymbol{i}}$. If a contiguous one-dimensional stencil for $\mathcal{P}_{\boldsymbol{i}}$ is defined by its left-most index $i_{m}$, then the first-order choice is clearly $i_{1}=i$. Then, for $k=2, \ldots, m$,

$$
i_{k}= \begin{cases}i_{k-1}-1, & \text { if }\left|\delta_{i_{k}-1}^{k}\right|<\left|\delta_{i}^{k}\right| \\ i_{k-1}, & \text { otherwise }\end{cases}
$$

Because this algorithm allows the reconstruction stencil to shift freely with the detection of any numerical gradient, it will be referred to as "freely adaptive." Reasons for modifying this search will become apparent.

A few observations that concern the available types of reconstruction operators are in order. Within the Shu-Osher approach, when conservation is desired, the implementation of the high-order reconstruction operator requires a uniform computational mesh. Therefore, the application of the ENO-FD algorithm on a non-uniform physical domain requires a sufficiently smooth transformation to a uniform mesh if third-order or higher accuracy is desired. An analogous form of this transformational reconstruction $(T R)$ can also be implemented within the finite-volume formulation. In this case, the reconstruction operator is applied to a set of volume-weighted averages $\left\{V_{i} \bar{U}_{i}\right\}$, and therefore sufficient smoothness in a spatial transformation is required. ${ }^{5}$ However, another option exists for the ENO-FV algorithm. The polynomial approximation can be performed in physical space, which releases such burdensome restrictions on grid smoothness. ${ }^{6,7,8}$ This latter procedure will be referred to as physical reconstruction $(P R)$. Although this $P R$ operator poses no problems in one dimension, its implementation in multidimensional space can be quite complex when the implementation must allow for local stencil adaptation. ${ }^{7,8}$ However, the multidimensional, finite-volume $T R$ operator can be readily implemented because it is defined as a product of one-dimensional operators. ${ }^{5}$ When required, the finite-volume algorithms will be distinguished as ENO-FV-TR and ENO-FV-PR.

\section{One-Dimensional Rarefaction Wave}

The first test case involves the solution of the Euler equations of gas dynamics in one spatial dimension, as it pertains to the movement of a right-traveling rarefaction 
wave through a domain of highly varying mesh spacing. The effects of two mesh transformations will be examined. One transformation has the required smoothness for a fourth-order $T R$ operator and the other does not.

For both transformations, the uniform computational domain is given by $\{-6 \leq \xi \leq 6\}$. The subset $\{-4 \leq \xi \leq 4\}$ is divided into five intervals: $[-4,-3],[-3,-1],[-1,1],[1,3]$, and $[3,4]$. In each of these interior intervals, the mapping $x=x(\xi)$ causes the physical mesh width $\Delta x$ to vary rapidly; the intervals $\{-6 \leq \xi<-4\}$ and $\{4<\xi \leq 6\}$ are mapped uniformly.

In the $k$-th interior interval, the first transformation is of the form

$$
x(\xi)=\xi_{k}+\sin \left[\frac{9 \pi}{20}\left(\xi-\xi_{k}\right)\right]
$$

where $\xi_{k}$ is an element of the set $\{-4,-2,0,2,4\}$. The mesh spacing $\Delta x$ in the uniform regions (fore and aft) is determined such that the connections at $\xi= \pm 4$ are smooth.

To generate a smoother grid on $\{-4 \leq \xi \leq 4\}$, a mapping of the following form is used:

$$
x(\xi)=\left[\alpha-\frac{5}{16} \beta\right] \xi+\frac{\beta}{32}\left[\frac{15}{\pi} \sin (\pi \xi)-\frac{3}{\pi} \sin (2 \pi \xi)+\frac{1}{3 \pi} \sin (3 \pi \xi)\right]
$$

The parameters $\alpha$ and $\beta$ are determined so that the ratio of the maximum to minimum values of $x_{\xi}$ are identical for the two grids, and that the physical distance between $x(-4)$ and $x(4)$ is the same for both.

Fig. 1(a) illustrates the similar behavior of these two transformations on $0<\xi<2$. The seemingly odd formula in Eq. (7) was chosen because its derivative is of the form

$$
x_{\xi}=\alpha-\beta \sin ^{6}\left(\frac{\pi}{2} \xi\right)
$$

The transformation derivatives are plotted on the same interval in Fig. 1(b). The value $\bar{x}_{\xi}$ represents the derivative normalized by its maximum value. For the transformation (6), $x_{\xi \xi}$ is discontinuous for $\xi= \pm 1, \pm 3$, which makes for an overall $C^{1}$ grid. The mesh produced by Eq. (7) will be $C^{\infty}$ on the nonuniform region and $C^{6}$ overall, because of the connections with the uniform intervals at $\xi= \pm 4$.

The initial solution consists of an isentropic expansion, smoothly distributed on $-6.5<x<-5.5$, with constant states, $U_{1}$ and $U_{2}$, on the right and left, respectively. The strength of this rarefaction is determined by requiring a mean temperature change of \pm 5 percent across the wave and supersonic flow on either side. The problem 
is nondimensionalized with respect to the mean solution. For $t>0$, the rarefaction wave moves to the right, and its behavior is monitored within the nonuniform region of the mesh until $t=4.0$.

Both algorithms used here are fourth-order accurate in the $L_{1}$ sense. Fig. 2 compares solutions for the two formulations by $x-t$ contour plots of the density, for $0.0<t<4.0$, on a mesh of 120 intervals with the $C^{1}$ transformation given by Eq. (6). The freely adaptive stencil algorithm in Eq. (5) is employed. All of the contour plots in this section represent the entire solution range, $\rho_{1}<\rho<\rho_{2}$, with 20 equally spaced levels. The ENO-FD solution exhibits significant qualitative error in neighborhoods about the second-derivative discontinuities in the mesh. In Fig. 2(b), such problems are not apparent when the solution is computed with the freely adaptive ENO-FV$\mathrm{PR}$ algorithm.

Fig. 3 depicts the use of the same fourth-order ENO-FD algorithm on the $C^{6}$ grid generated by Eq. (7). With the required mesh smoothness to support the reconstruction, no visible distortions appear as on the $C^{1}$ grid. However, the fourthorder design accuracy is not achieved for this solution when density error is measured with respect to the $L_{1}$ norm in a region to the left of the rarefaction at $t=4.0$. The same accuracy problem was found with the freely adaptive ENO-FV-PR scheme. The results of both mesh-refinement studies are shown on a log-log plot in Fig. 4(a). The spatial discretizations employed in this study are 120, 240, 480, and 960 intervals. The numbers on the loci represent the computational order of accuracy as measured between the two finest meshes.

A similar loss-of-accuracy phenomenon was reported by Rogerson and Meiberg ${ }^{9}$, which prompted a response from $\mathrm{Shu}^{10}$ that the problem arises by allowing the stencil to adapt too freely. Shu has suggested that the stencil adaptation algorithm be modified to bias the stencil towards one that is stable, in the sense of linear stability analysis. In the present application, the resulting stencil is one which is upwind biased. This biasing can be done by implementing a factor $\sigma$ in the stencil search in Eq. (5), vis.

$$
i_{k}= \begin{cases}i_{k-1}-1, & \text { if } \sigma_{\mathrm{L}}\left|\delta_{i_{k}-1}^{\mathrm{k}}\right|<\sigma_{\mathrm{R}}\left|\delta_{i_{k}}^{\mathrm{k}}\right| \\ i_{k-1}, & \text { otherwise }\end{cases}
$$

where $\left(\sigma_{L}, \sigma_{R}\right)=(1, \bar{\sigma})$ or $(\bar{\sigma}, 1)$, for biasing to the left or right, respectively, with $\bar{\sigma}>1$.

Fig. 4(b) shows grid-refinement results that are analogous to those in Fig. 4(a) using the modification in Eq. (8) with $\bar{\sigma}=2.0$; however, a fourth-order error reduction 
is still not evident because the error is measured in a region where numerical gradients are extremely small and ratios of neighboring gradients may be much larger than the chosen biasing parameter. Atkins ${ }^{11}$ has suggested that an additional constraint on the stencil adaptation is required. The purpose of this constraint is to bias the stencil toward one that is stable wherever the solution is smooth. A parameter installed for this purpose can be considered a lower threshold for the magnitudes of the local differences $\left\{\delta_{j}^{k}\right\}$, below which a stencil is forced toward a stable target, regardless of the relative magnitudes of neighboring numerical gradients. The present implementation of such a parameter can be written

$$
\begin{gathered}
\text { if }\left|\delta_{i_{k}-1}^{k}\right|<\epsilon \text { and }\left|\delta_{i_{k}}^{k}\right|<\epsilon \\
\text { then } \quad i_{k}=i_{k}^{\star},
\end{gathered}
$$

where $\epsilon$ is a small parameter, and $i_{k}^{\star}$ defines the stable stencil at the $k$-th level.

Another grid-refinement study was performed, with Eqs. (8) and (9) wherein $\bar{\sigma}=2.0$ and $\epsilon=0.01$. The results are shown in Fig. 5(a) and are much more consistent with the scheme's design accuracy. In fact, the ENO-FV-PR algorithm, coupled with the stenciling modifications in Eqs. (8) and (9), yields fourth-order computational accuracy on the $C^{1}$ grid, which is shown in Fig. 5(b). The second-order convergence exhibited by the ENO-FD algorithm in this plot is expected. However, Fig. 6 shows that the qualitative error in the ENO-FD solution on the $C^{1}$ grid, that was previously shown in Fig. 2(a), has been significantly reduced with these stencil biasing modifications.

At this point, the apparently greater robustness of the ENO-FV algorithm can be attributed to the fact that this formulation allows for a physical reconstruction that is not available for the finite-difference algorithm. However, as previously noted, multidimensional extensions for this more generalized operator can be complicated and costly. Therefore, some results produced with the ENO-FV-TR algorithm will be discussed. In Fig. 7(a), when the freely adaptive version of this algorithm is implemented on the $C^{6}$ grid, significant error exists that was not evident in the analogous case for the ENO-FD solution in Fig. 3. This error is due to the reconstruction of volume-weighted averages, in which a rapidly varying mesh will have an inordinate effect on the stencil choice in a region of small solution gradients. However, biasing the reconstruction stencils with Eqs. (8) and (9) is not enough to entirely rid the solution of these grid distortion errors, as shown in Fig. 7(b). The problem is still 
related to the reconstruction of volume-weighted averages, in particular to the fact that a uniform flow is not preserved by this procedure if the analytic transformation is used for the necessary discrete values $\left\{x_{\xi_{i}}\right\}$. Therefore, instead of using Eq. (7b) to compute the derivatives, these derivatives are numerically approximated in a manner that enables the reconstruction operator to preserve the free stream exactly ${ }^{5}$. The resulting solution, with this final modification, is shown in Fig. 7(c). In this final form, the numerical accuracy of the ENO-FV-TR algorithm is found to perform to design as shown in Fig. 8(a). The ENO-FD and ENO-FV-PR results from Fig. 5(a) are repeated in Fig. 8(b), for comparison.

\section{Two-Dimensional Channel Flow}

The ENO-FD and ENO-FV algorithms are now compared in two spatial dimensions. The test case involves a steady, subsonic flow in a channel of varying area. Although high-order ENO schemes are clearly designed with unsteady solutions in mind, many such solutions of interest can be considered as the imposition of perturbations upon a steady flow. It is therefore essential that a steady flow be accurately predicted in order to obtain meaningful results from unsteady problems of an aeroacoustic or transitional nature. Unless otherwise stated, the remainder of the applications of the ENO-FV algorithm will involve the $T R$ type of reconstruction.

This channel flow solution is assumed to be governed by the two-dimensional Euler equations and is computed with both the ENO-FD and ENO-FV algorithms on two different geometries. For the two channels under consideration, the lengthto-height ratio is $L / H=1.5$, with constant-area sections fore and aft of a section of varying area. Each constant-area section has length $L / 5$; at the throat, the constriction is 10 percent of $H$. The most significant difference between the two geometries is the order of smoothness to which the constant-area sections are connected to the center section. Both channels can be described as follows. Let the rectangle $\{0 \leq \xi \leq L\} \times\{0 \leq \eta \leq H\}$ denote the computational domain for each channel. The identity maps $(\xi, \eta)$ to the physical point $(x, y)$, for $\xi$ in $[0, L / 5] \cup[4 L / 5, L]$ and all $\eta$. On the interval $\{L / 5<\xi<4 L / 5\}$, the geometry for the varying-area section is given by a transformation of the form

$$
\begin{aligned}
& x=\xi \\
& y=\frac{\eta}{H} y_{2}(\xi)+\left(1-\frac{\eta}{H}\right) y_{1}(\xi)
\end{aligned}
$$

where $y_{1}(\xi)$ and $y_{2}(\xi)$ are, respectively, the equations for the bottom and top walls. 
The first middle section considered is determined by walls of the form

$$
y_{i}(\xi)=a_{i} \sin ^{4}\left(b_{i} \xi+c_{i}\right)+d_{i}, \quad i=1,2
$$

where the values of the coefficients are set for the desired throat constriction and a smooth connection to the constant-area sections. Three continuous $\xi$ derivatives of $y$ exist at $\xi=L / 5,4 L / 5$, for all $\eta$. Therefore, Eqs. (10) and (11) generate a $C^{3}$ geometry. Fig. 9 illustrates this geometry on a $60 \times 40$ mesh. The second channel geometry differs from the first only in that the walls in the middle section are given by polynomials of the form

$$
y_{i}(\xi)=a_{i}+b_{i} \xi^{2}+c_{i} \xi^{4}, \quad i=1,2
$$

In this case, the connections of the middle to the outer sections are continuous in $\xi$ to only one derivative, for $0<\eta<H$.

The desired solution is that of a steady-state flow that is caused by a uniform, parallel free stream entering the channel at $x=0$. This solution is achieved by solving the time-dependent Euler equations with the fourth-order algorithms. At $t=0$, the solution in the entire channel is set to free stream with a Mach number of 0.3 . Tangency is imposed on the walls, and a non-reflecting boundary condition ${ }^{12}$ is applied at the inflow and outflow. The stencil-biasing modifications in Eqs. (8) and (9) are essential for convergence of the solution to a steady state, and are implemented with $\bar{\sigma}=2.0$ and $\epsilon=0.01$. Flux residuals were readily driven to machine zero in both test cases.

These solutions were computed by both algorithms on a sequence of successively refined grids, and the solutions' errors were determined by deviation from isentropy, as measured by the quantity $S=P / \rho^{\gamma}$. The refinement sequence employed here is $15 \times 10,30 \times 20,45 \times 30,60 \times 40$, and $90 \times 60$. The results of the grid-refinement study on the $C^{3}$ geometry are shown in Figs. 10(a), (b), and (c). The "Global" error is computed over the entire computational domain, the "Wall" error is computed only at the points along one wall, and the "Interior" error is computed on $\{L / 4<\xi<3 L / 4\} \cup\{H / 4<\eta<3 H / 4\}$. Both algorithms evidently perform at or near design accuracy, as expected for fourth-order schemes on a $C^{3}$ mesh.

In Figs. 10(d), (e), and (f) are the grid refinement results for the $C^{1}$ mesh. As expected, second-order results are obtained on the wall for both algorithms. However, the finite-volume algorithm performs at third-order accuracy with respect 
to the global error and at design accuracy on the interior, while the finite-difference algorithm shows second-order accuracy in all three measures. These results suggest that, for the ENO-FD algorithm, the second-order entropy error that arises from the non-smoothness at the section connections is propagating into the interior. This assumption is supported by Fig. 11, in which the quantity $\log S$ is plotted along the center line of a $60 \times 40$ mesh. Clearly, in the finite-difference solution, there is a lower-order entropy error within the middle section than exists in the constant-area sections.

\section{Oblique Sod's Problem}

The final test under consideration is selected to compare the capabilities of the two algorithms to resolve waves that are oblique to the computational mesh. Sod's problem $^{13}$ will be solved in two-dimensional space so that the planar waves produced will propagate at various angles of incidence with respect to a rectangular grid. The intent is not only to inspect the qualitative resolution of the oblique waves, but also to quantify the manner in which each algorithm detects an oblique wave with respect to its detection of a wave that is normal to the mesh.

Sod's problem is a Riemann problem which is subject to the solution of the Euler equations and determined by initial conditions that consist of a thermodynamic discontinuity imposed upon a fluid at rest. The magnitudes of the density and pressure jumps are, from left to right, $\rho_{L} / \rho_{R}=8$ and $P_{L} / P_{R}=10$, respectively. Such an initial solution is installed on a two-dimensional Cartesian grid so that the discontinuity is a straight line that makes an angle $\theta$ with the $x$ axis. The physical mesh varies with $\theta$ in the following manner. A computational mesh is defined on the rectangle $[0, L] \times[0, H]$, with a length-to-height ratio $L / H=6$. If $0<\theta \leq \pi / 2$, then the physical domain is $\left\{0<x<L_{\theta}\right\} \times\left\{0<y<H_{\theta}\right\}$, and is determined by $H_{\theta}=H / \sin \theta$ and $L_{\theta}=L / \sin \theta$. This scaling achieves the same grid resolution normal to the wave propagation on a given mesh at some fixed time for all choices of $\theta$. The mesh discretization in each direction is chosen such that $\Delta x=\Delta y$.

At $t=0$, the initial discontinuity is positioned at $(x, y)=(3 L / 8,0)$ and inclined at the angle $\theta$. The angles of inclination are chosen so that $\tan \theta$ is an integer and that the boundary conditions at $y=0$ and $y=H_{\theta}$ can be determined in a "shiftedperiodic" manner. In particular, the test angles are $\theta=\arctan 1, \arctan 2, \operatorname{and} \arctan 4$, in addition to the one-dimensional problem $\theta=\pi / 2$. The solution is computed to $t=1.2$ The stencil biasing in Eq. (8) is used in the reconstruction procedures with 
$\bar{\sigma}=2.0$.

Fig. 12(a) represents the solution at $t=1.2$, on a $96 \times 16$ grid, with $\theta=\arctan 1$, with the use of the ENO-FV algorithm. The three wave structures are, from left to right, an expansion wave, a contact discontinuity, and a shock. The axis variables $x^{\prime}$ and $y^{\prime}$ represent the physical coordinates scaled by $\sin \theta$. Fig. 12(b) depicts the same solution as in Fig. 12(a), for both algorithms, along the grid line $y^{\prime}=0$. On this level, the qualitative difference between the solutions is barely detectable. Therefore, in Fig. 13, the solutions are examined in the vicinity of the contact discontinuity for all test values of $\theta$. (The successive loci in this plot have been shifted upward and to the right.) Although the ENO-FV scheme produces a slightly steeper numerical gradient at the location of the discontinuity, such a small difference can be judged insignificant. Fig. 13, then, suggests that both algorithms perform equally well with respect to oblique waves irrespective of $\theta$. On a more quantitative level, the differences between the oblique cases and the one-dimensional case, for each algorithm, have been measured. These results are plotted in Figs. 14(a) and 14(b). By this measure, the finite-volume scheme performs marginally better, particularly with respect to the rarefaction wave.

\section{Cost Comparison}

As with any numerical algorithm, the cost of implementing either the ENO-FD or ENO-FV scheme is a major concern. Conceptually, the two formulations can be made equally cost effective for high-order solutions of one dimensional problems or for first or second-order accurate solutions in multiple dimensions. However, for third or higher-order accuracy in two or more dimensions, the algorithms are radically different. This difference translates to a significant disparity in cost.

With regard to cost, the ENO-FD algorithm has a clear advantage when applied to multidimensional problems. This advantage can be entirely attributed to the fact that the finite-difference operator solves a system of equations in a pointwise manner. In this case, a high-order multidimensional reconstruction can be accomplished in a dimension-by-dimension fashion. If the algorithm is $r$-th order accurate in the $L_{1}$ sense, then the reconstruction stencil in $k$ dimensions contains $k(r-1)+1$ points. The dimension-by-dimension approach to reconstruction is not possible within the finite-volume formulation, if third-order or better accuracy is required. Because the solution at any time is in the form of cell averages, the multidimensional ENO-FVTR reconstruction is implemented as a product of one-dimensional operators, and 
thereby requires $r^{k}$ cells in a reconstruction stencil. Moreover, the Gaussian quadrature used to integrate the flux on each cell boundary requires multiple solutions of the Riemann problem on each cell interface. The number of points $n_{q}$ required in this quadrature on an interface is $n_{q}=[\operatorname{int}((r+1) / 2)]^{k-1}$, where int $(\cdot)$ denotes integer truncation. This high-order quadrature is entirely avoided within the finitedifference approach. It should be noted that both formulations require the same amount of logic in the stencil adaptation, which is the most expensive part of either algorithm.

Fig. 15 depicts the relative CPU cost of the two algorithms, for solutions of the Euler equations in two dimensions, for 2nd, 3rd, 4th, and 5th-order accurate applications. CPU times were measured on a Cray-YMP and normalized with respect to the 2nd-order ENO-FD cost. An estimated cost comparison for threedimensional problems can be obtained by the following reasoning. For a specified order of accuracy, let $C_{1}$ denote the cost of solving one equation at a point in one dimension; this is the same for either algorithm. Then, because of its dimension-bydimension implementation, the estimated cost incurred by the ENO-FD algorithm in solving the Euler equations in $k$ dimensions on a grid of $N^{k}$ points is

$$
C_{F D}=C_{1}(k+2) k N^{k}
$$

This formula predicts a cost-per-point increase factor of $8 / 3$ when the one-dimensional ENO-FD algorithm is extended to two dimensions. This value has been supported by computer measurements. The corresponding factor for the extension from two to three dimensions is $15 / 8$. The estimation of a three-dimensional cost for the ENOFV algorithm cannot be done by such a simple linear extrapolation. There are two significant costs in addition to the base value given by Eq. (13). One is the extension of the finite-volume reconstruction to a three-dimensional product. The other arises from the additional $3\left(n_{q}-1\right)$ flux computations that are required in the integration over the surface of each cell. These additional costs were extrapolated from twodimensional CPU measurements on a Cray-YMP. Fig. 16 represents the estimated three-dimensional cost comparison where, again, the data are normalized by the 2nd-order cost.

\section{Concluding Remarks}

For accuracy on a sufficiently smooth mesh and resolution of oblique waves, both algorithms perform equally well. The finite-volume implementation is less sensitive 
to derivative discontinuities, whether in the computational mesh or in the solution. In particular, the ENO-FV-PR algorithm has the capacity to perform at design accuracy, independent of the mesh. Although the generalized multidimensional adaptive-stenciling implementation of this algorithm has not sufficiently matured, some promising results can be found in Ref. 8. Either of the multidimensional finitevolume algorithms is significantly more costly than the finite-difference algorithm. Therefore, if, for a given application, the computational domain is known to be sufficiently smooth and can be suitably structured, then the ENO-FD algorithm is the method of choice. However, for problems with complex geometries, it might pay to use the more expensive algorithm if the grid is significantly less costly to generate in a less restrictive fashion. 


\section{References}

${ }^{1}$ Harten, A., Engquist, B., Osher, S., and Chakravarthy, S., "Uniformly High Order Accurate Essentially Non-oscillatory Schemes III," Journal of Computational Physics, Vol. 71, No. 2, 1987, pp. 231-323.

${ }^{2}$ Shu, C. and Osher, S., "Efficient Implementation of Essentially Non-Oscillatory Shock-Capturing Schemes," Journal of Computational Physics, Vol. 77, No. 2, 1988, pp. $439-471$.

${ }^{3}$ Harten, A., "Preliminary Results on the Extension of ENO Schemes to TwoDimensional Problems," in Proceedings, International Conference on Nonlinear Hyperbolic Problems, Saint-Etienne, 1986, Lecture Notes in Mathematics, edited by C. Carasso et al. (Berlin, 1987), p. 23.

${ }^{4}$ Shu, C. and Osher, S., "Efficient Implementation of Essentially Non-Oscillatory Shock-Capturing Schemes II," Journal of Computational Physics, Vol. 83, (1989), p. 32.

${ }^{5}$ Casper, J. and Atkins, H., "A Finite-Volume High-Order ENO Scheme for TwoDimensional Hyperbolic Systems," Journal of Computational Physics, Vol 106, May, 1993, pp 62-76.

${ }^{6}$ Barth, T. J. and Frederickson, P. O. "High-Order Solution of the Euler Equations on Unstructured Grids Using Quadratic Reconstruction," AIAA 90-0013, January, 1990.

${ }^{7}$ Harten, A. and Chakravarthy, S., "Multi-Dimensional ENO Schemes for General Geometries," NASA Contractor Report 187637, ICASE Report No. 91-76, September, 1991.

${ }^{8}$ Abgrall , R., "Design of an Essentially Non-Oscillatory Reconstruction Procedure on Finite-Element Type Meshes," NASA Contractor Report 189574, ICASE Report No. 91-84, December, 1991.

${ }^{9}$ Rogerson, A. M. and Meiberg, E., "A Numerical Study of the Convergence Properties of ENO Schemes," Journal of Scientific Computing, Vol. 5, No. 2, 1990, pp. 151-167.

${ }^{10} \mathrm{Shu}, \mathrm{C}$. ." "Numerical Experiments on the Accuracy of ENO and Modified ENO Schemes," Journal of Scientific Computing, Vol. 5, No. 2, 1990, pp. 127-150.

${ }^{11}$ Atkins, H., "High-Order ENO Methods for the Unsteady Compressible NavierStokes Equations," AIAA 91-1557, AIAA 10th Computational Fluid Dynamics Con- 
ference, June 24-26, 1991.

${ }^{12}$ Atkins, H. and Casper, J. "Non-Reflective Boundary Conditions for High-Order Methods," AIAA 93-0152, AIAA 31st Aerospace Sciences Meeting, Reno, Nevada, January, 1993.

${ }^{13}$ Sod, G. A., "A Survey of Several Finite-Difference Methods for Systems of Nonlinear Hyperbolic Conservation Laws," Journal Computational Physics, Vol. 27, 1978, pp. $1-31$. 

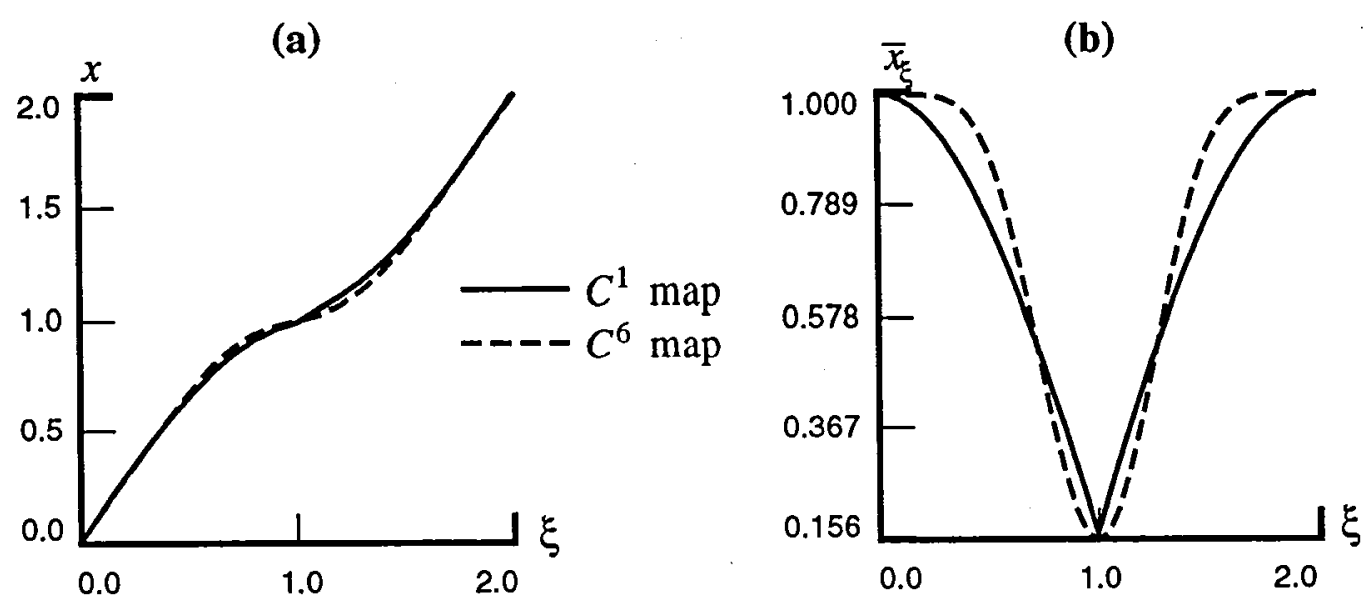

Fig. 1. (a) The transformations of Eqs. (6) and (7). (b) Normalized derivatives of Eqs. (6) and (7).

(a)

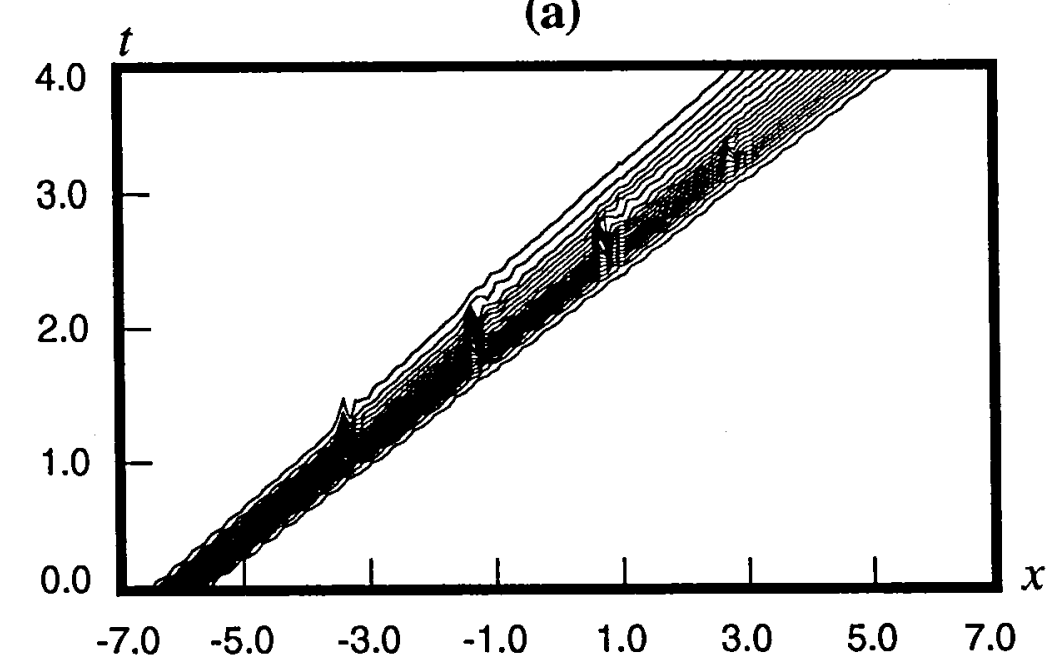

(b)

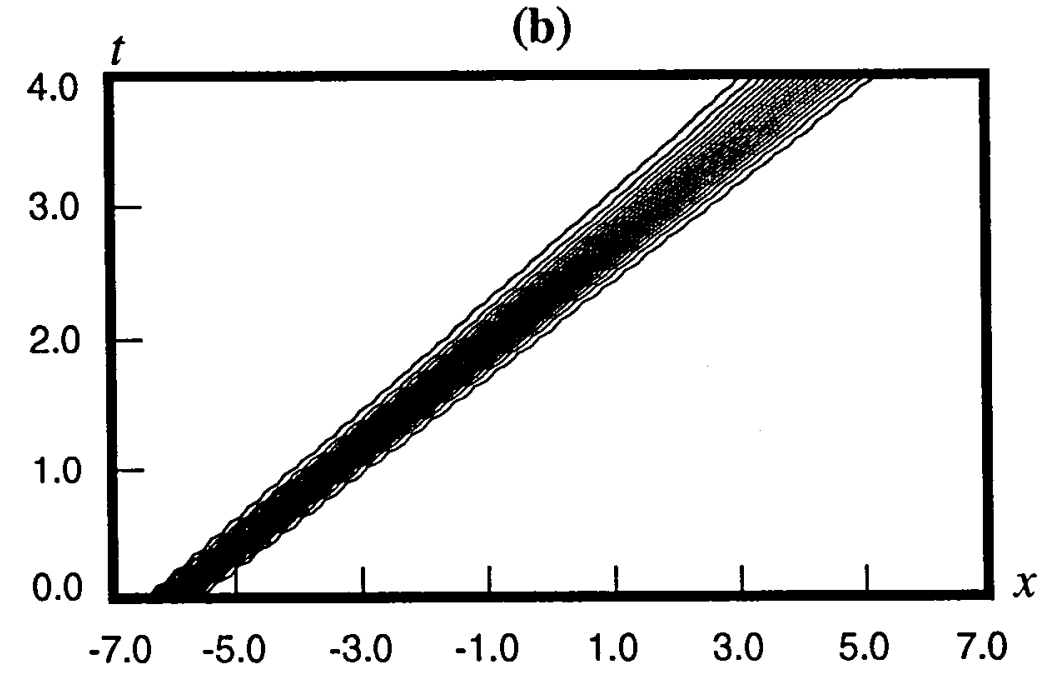

Fig. 2. Density, on the $C^{1}$ mesh. (a) ENO-FD, freely adaptive stencil. (b) ENO-FV-PR, freely adaptive stencil. 


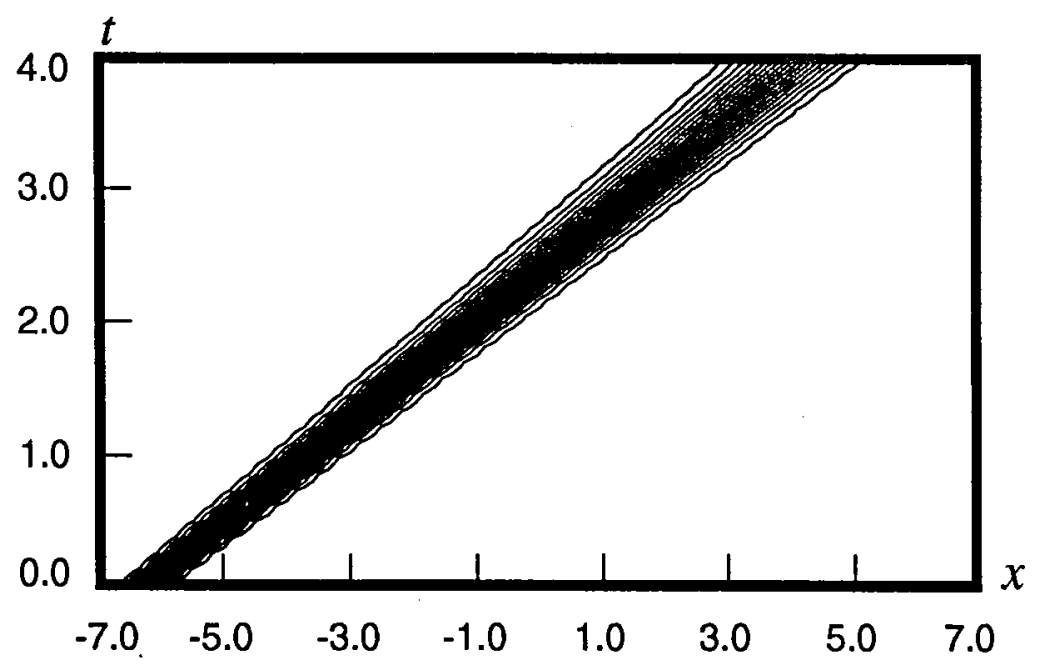

Fig. 3. Density, on the $C^{6}$ mesh, ENO-FD, freely adaptive stencil.

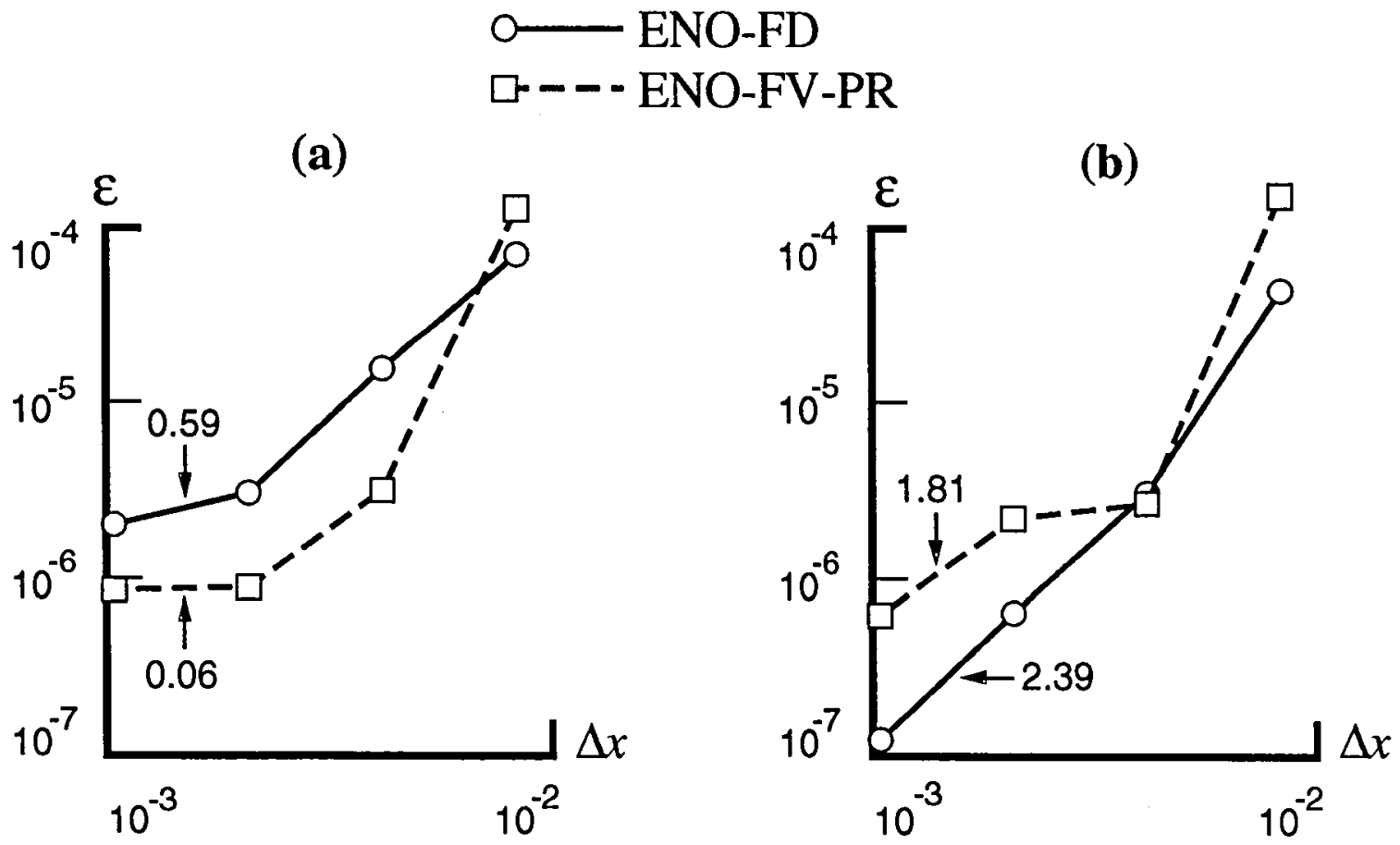

Fig. 4. $L_{1}$ density error, $t=4.0, C^{6}$ grid. (a) Freely adaptive stencil. (b) Biased stencil $(\bar{\sigma}=2.0)$. 

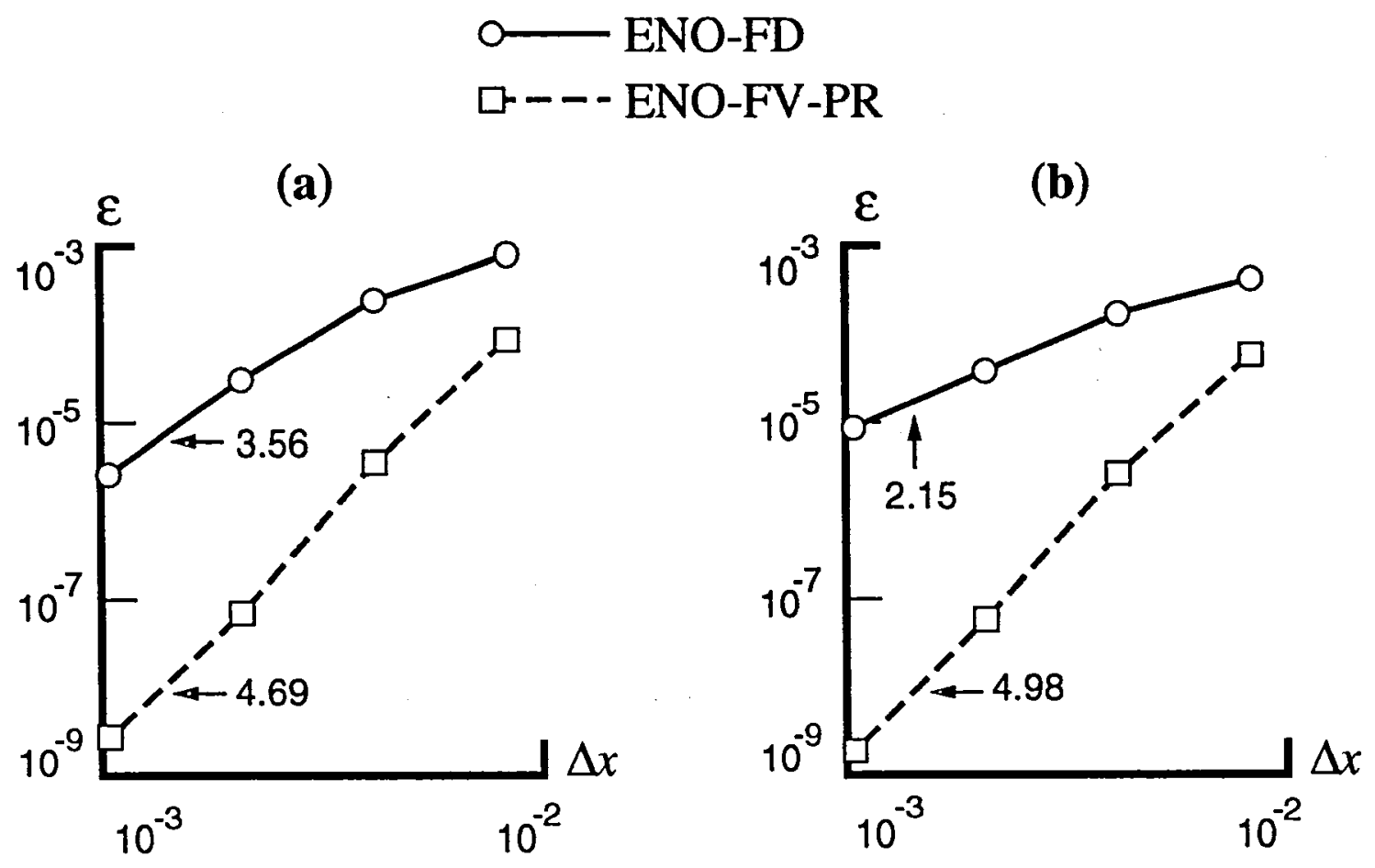

Fig. 5. $L_{1}$ density error, $t=4.0$. (a) $C^{6}$ grid, biased stencil $(\bar{\sigma}=2.0, \epsilon=0.01)$. (b) $C^{1}$ grid, biased stencil $(\bar{\sigma}=2.0, \epsilon=0.01)$.

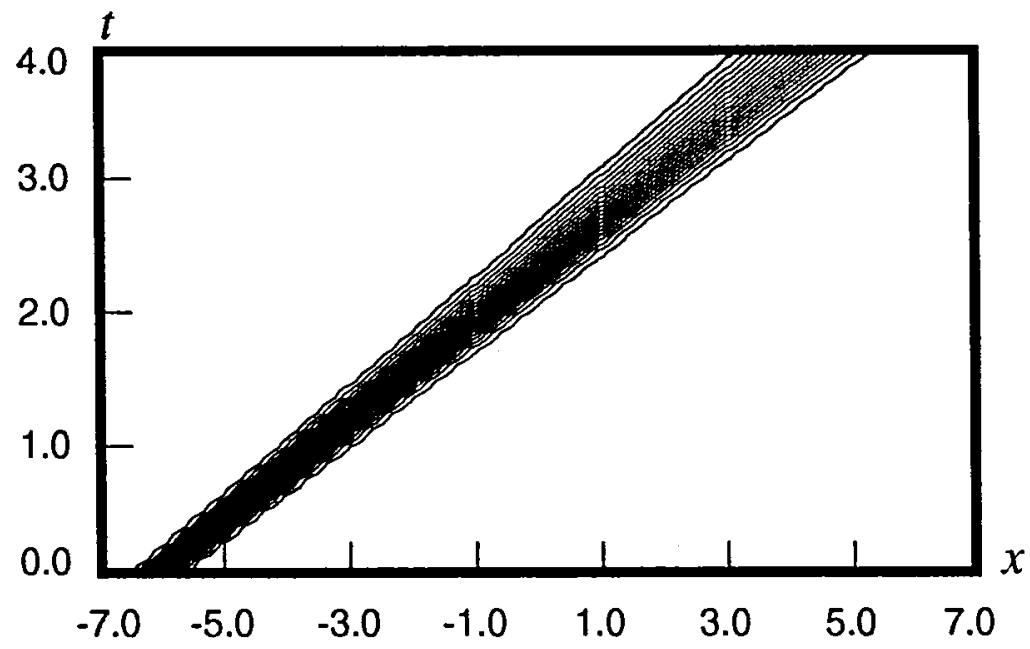

Fig. 6. Density, on the $C^{1}$ mesh, ENO-FD, biased stencil $(\bar{\sigma}=2.0, \epsilon=0.01)$. 
(a)

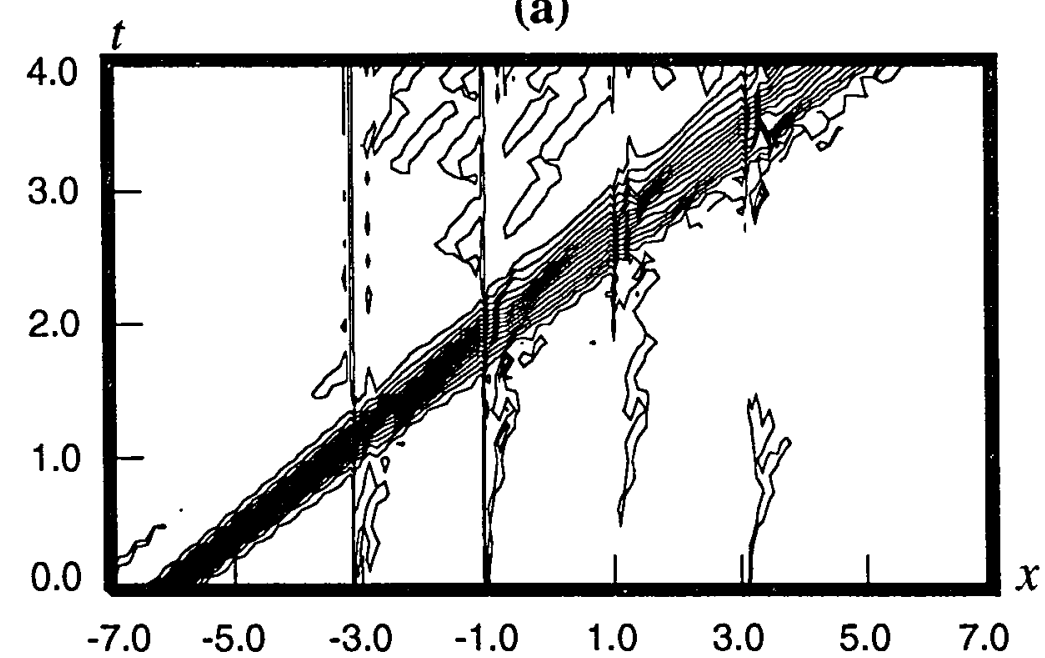

(b)

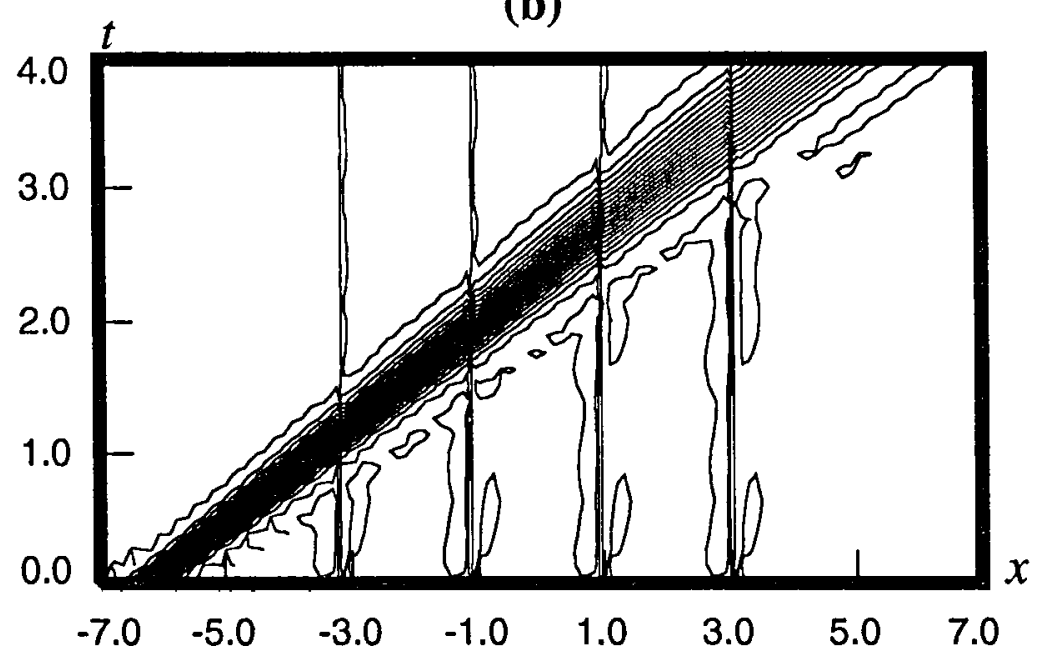

(c)

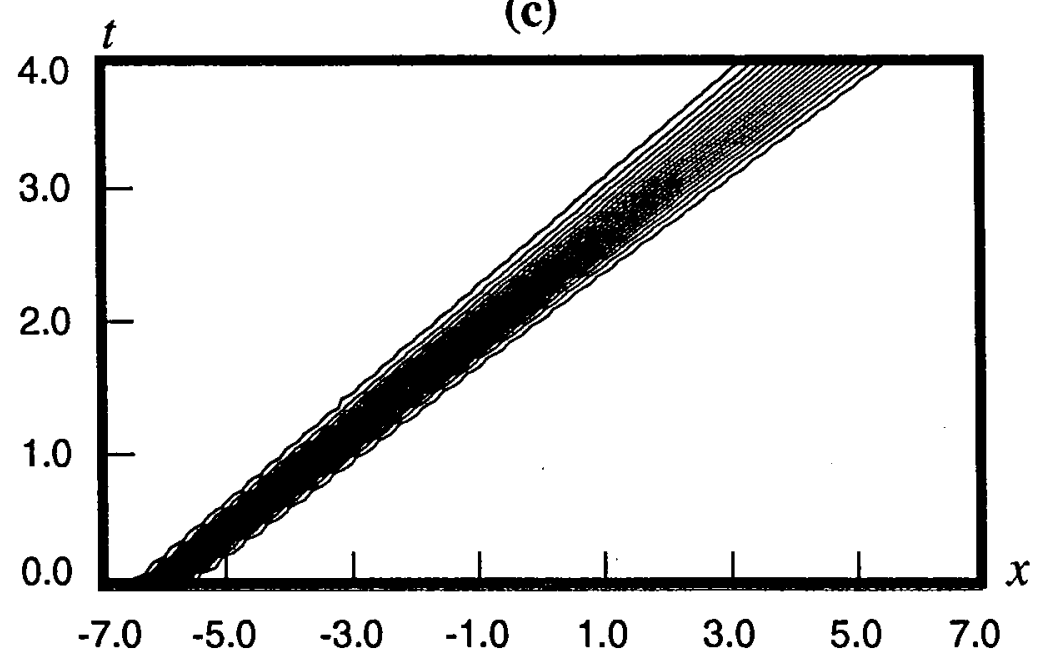

Fig. 7. Density, on the $C^{6}$ mesh, ENO-FV-TR. (a) Freely adaptive stencil. (b) Biased stencil ( $\bar{\sigma}=2.0, \epsilon=0.01$ ). (c) Biased stencil, free-stream preserving. 

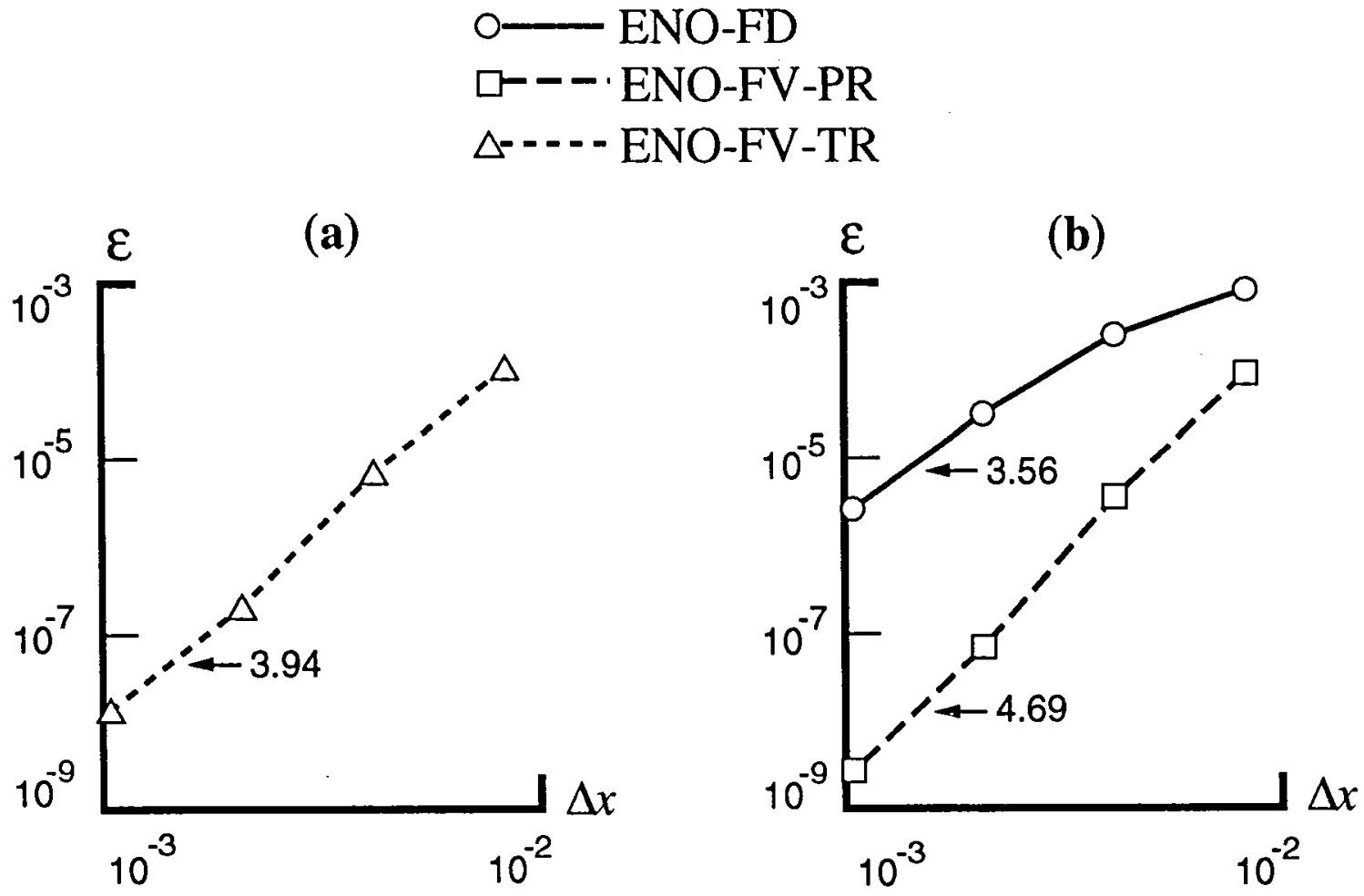

Fig. 8. $L_{1}$ density error, $t=4.0, C^{6}$ grid. (a) Biased stencil, free-stream preserving. (b) Biased stencil $(\bar{\sigma}=2.0, \epsilon=0.01)$.

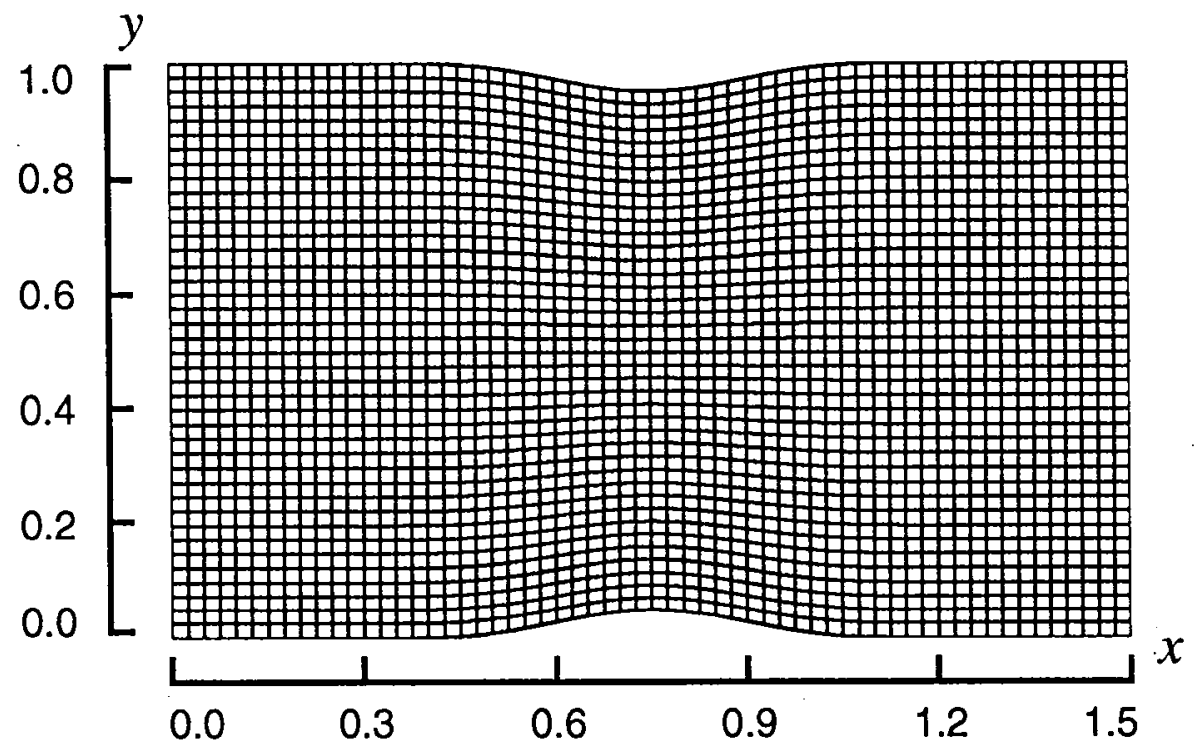

Fig. 9. The $C^{3}$ geometry of Eqs. (10) and (11). 

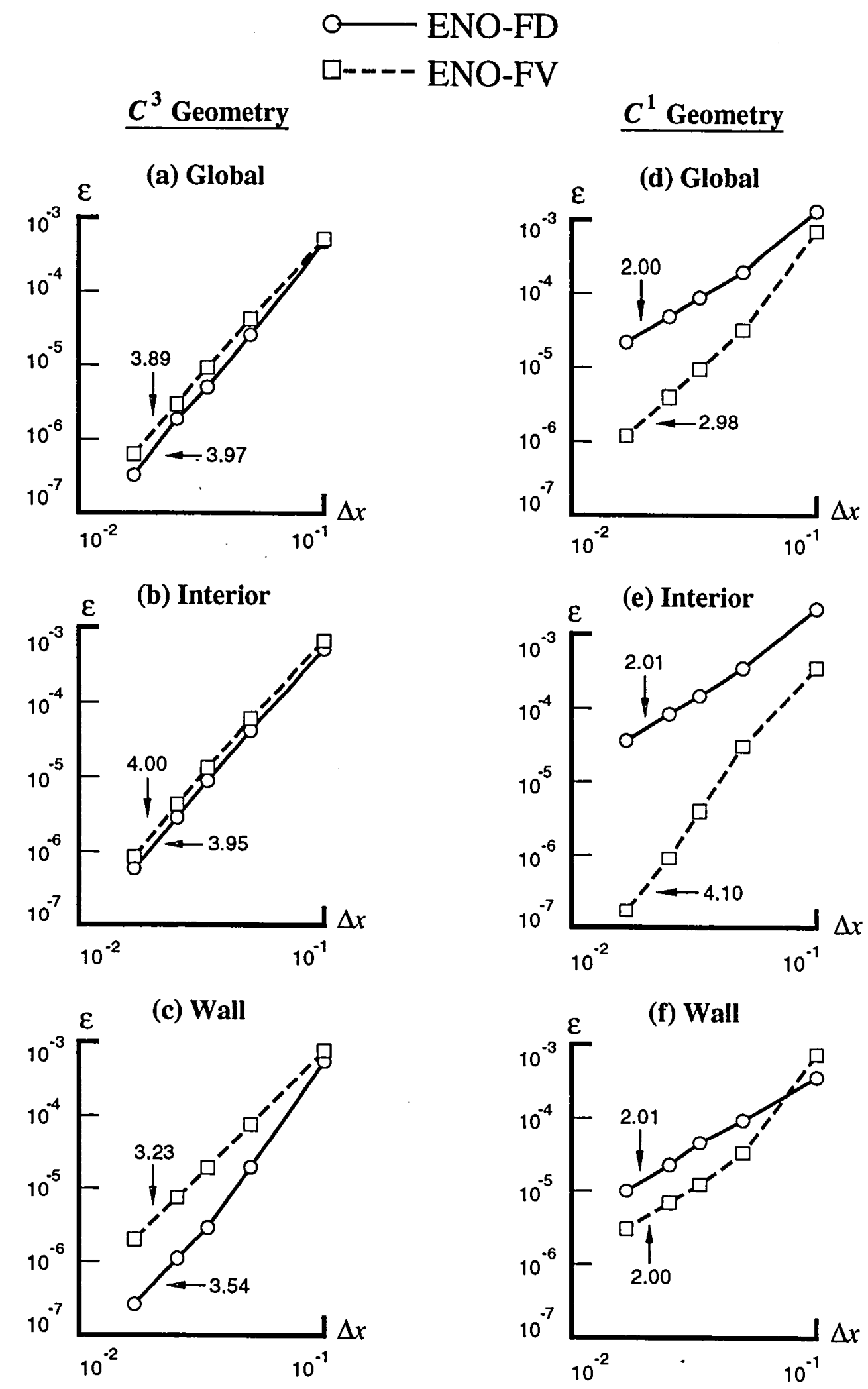

Fig. 10. $L_{1}$ entropy errors, steady $2 \mathrm{D}$ channel flow. 


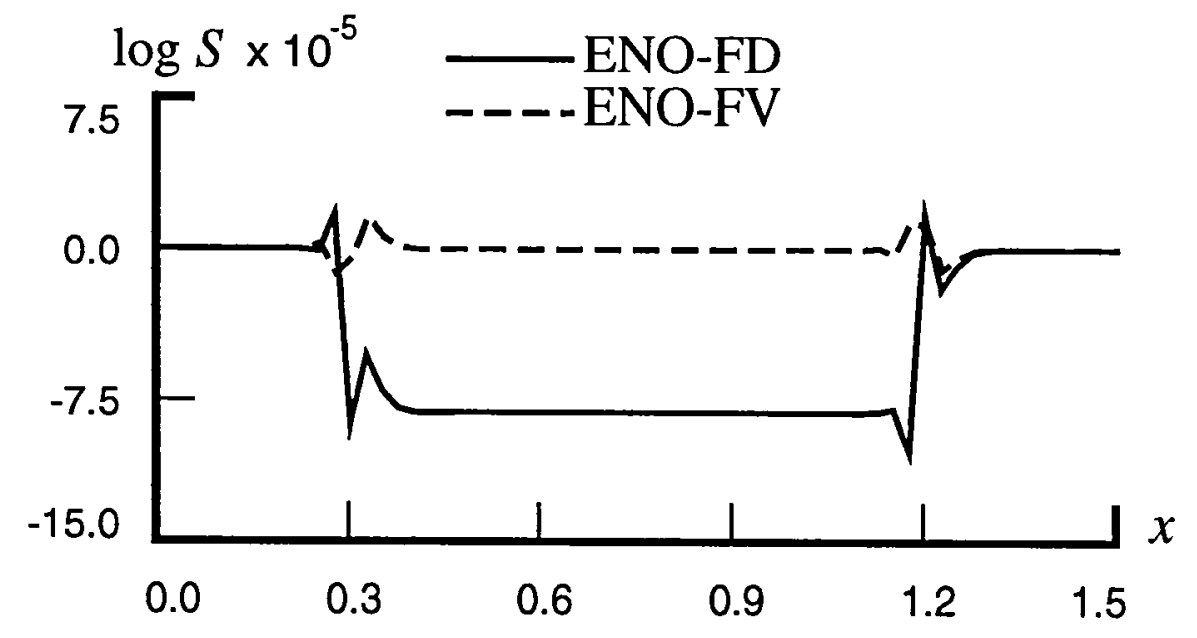

Fig. 11. Center-line entropy, $C^{1}$ grid.
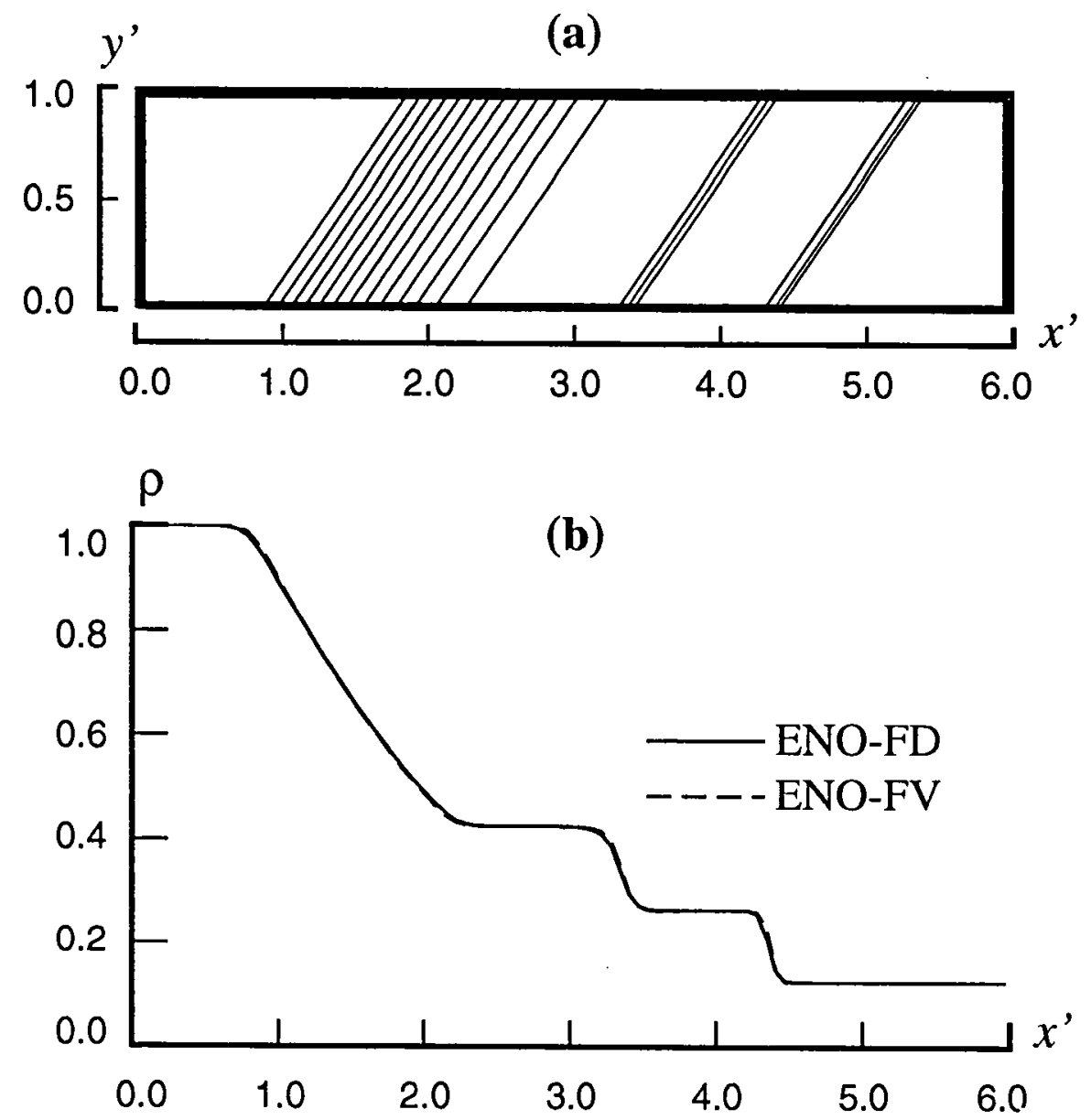

Fig. 12. (a) Density contours, ENO-FV, $\theta=\arctan 1$. (b) Density, $y^{\prime}=0.0, \theta=\arctan 1$. 


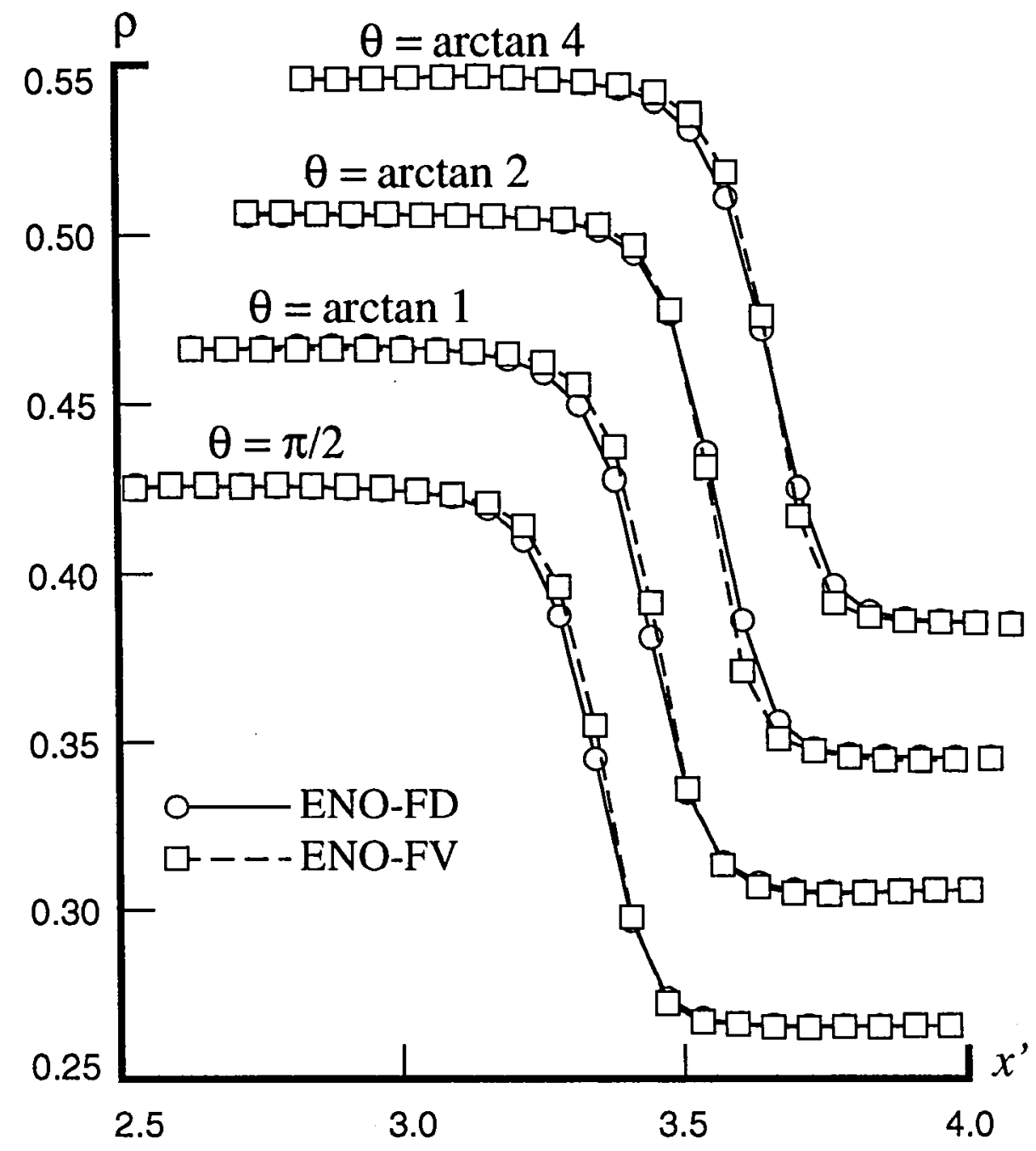

Fig. 13. Density, contact discontinuity, $y^{\prime}=0.0$. 

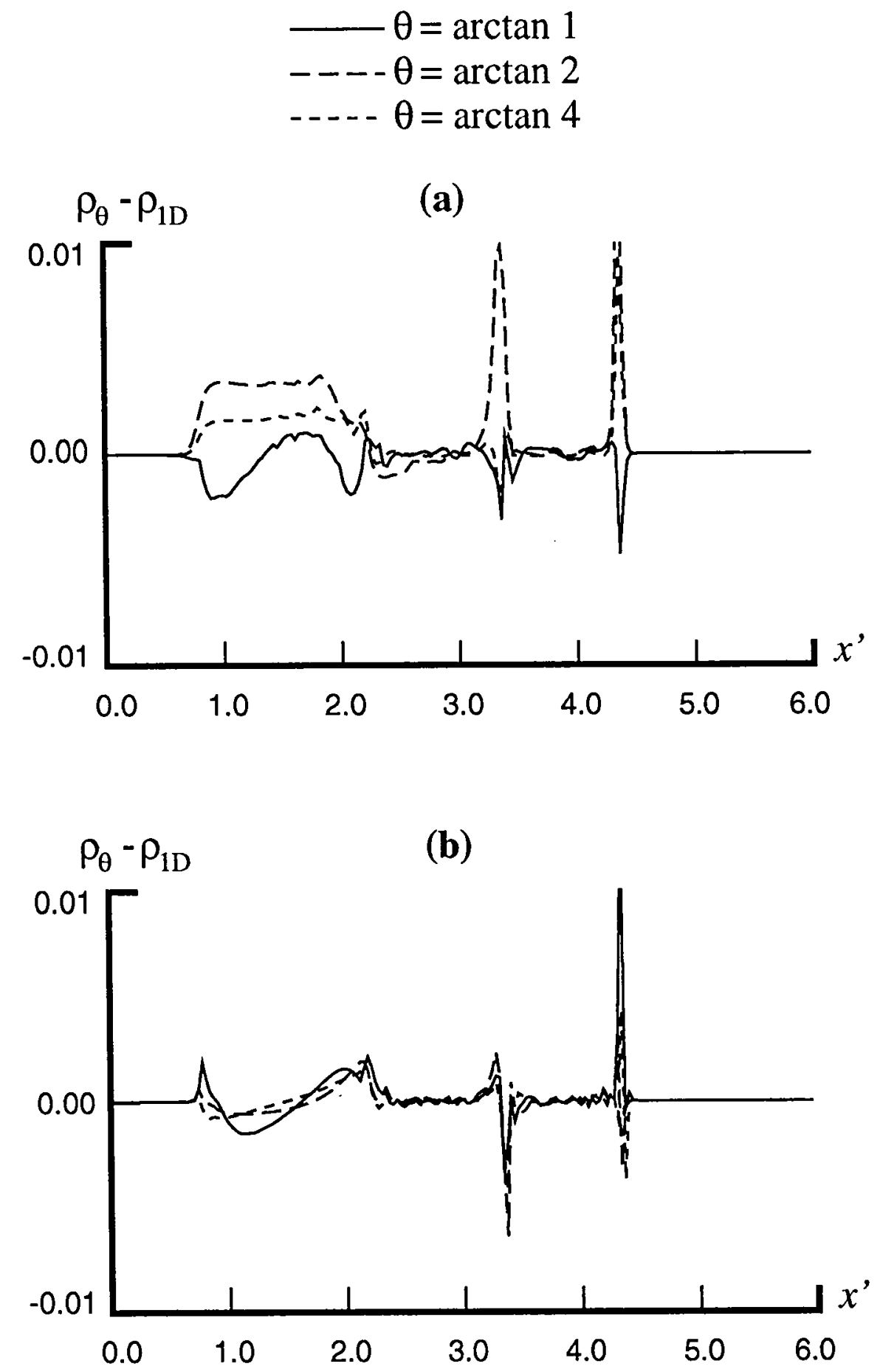

Fig. 14. (a) Density deviation from 1D, ENO-FD. (b) Density deviation from 1D, ENO-FV. 


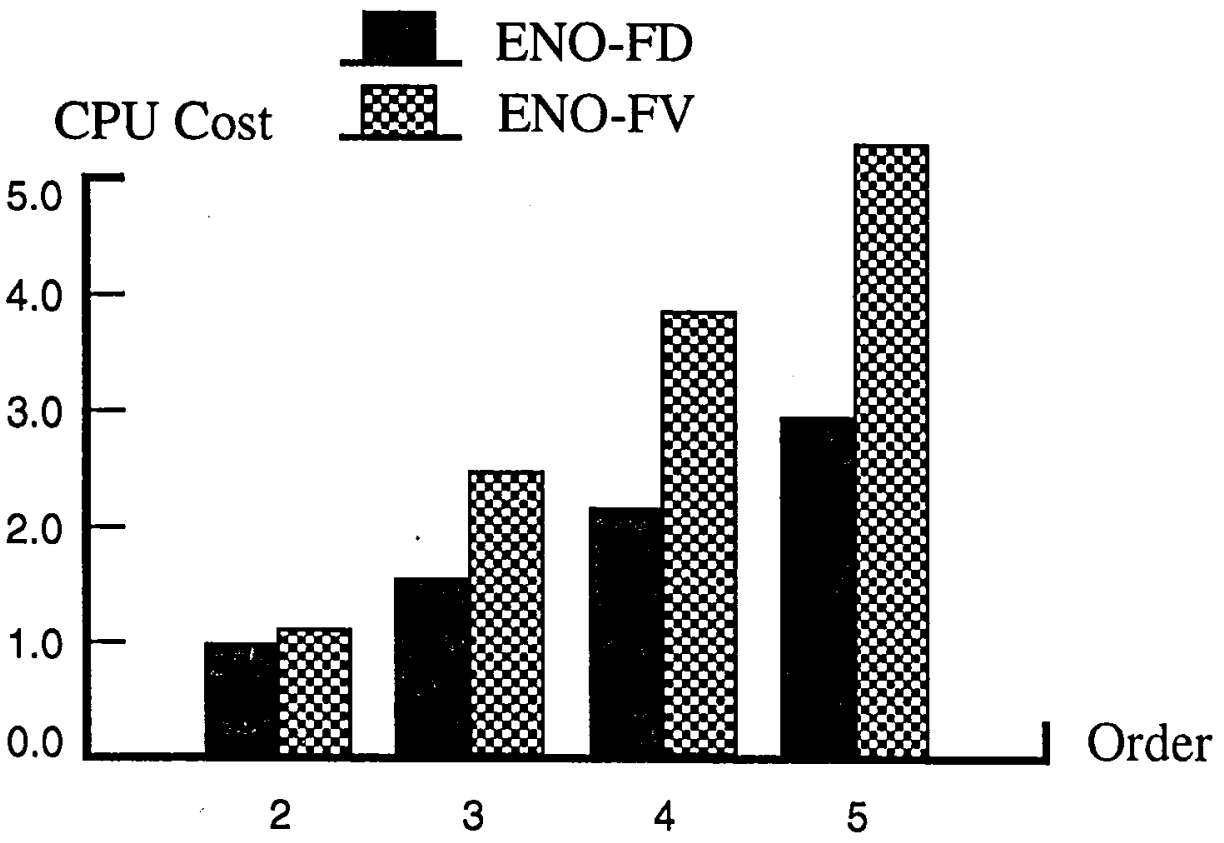

Fig. 15. Relative CPU cost of the two algorithms in two spatial dimensions.
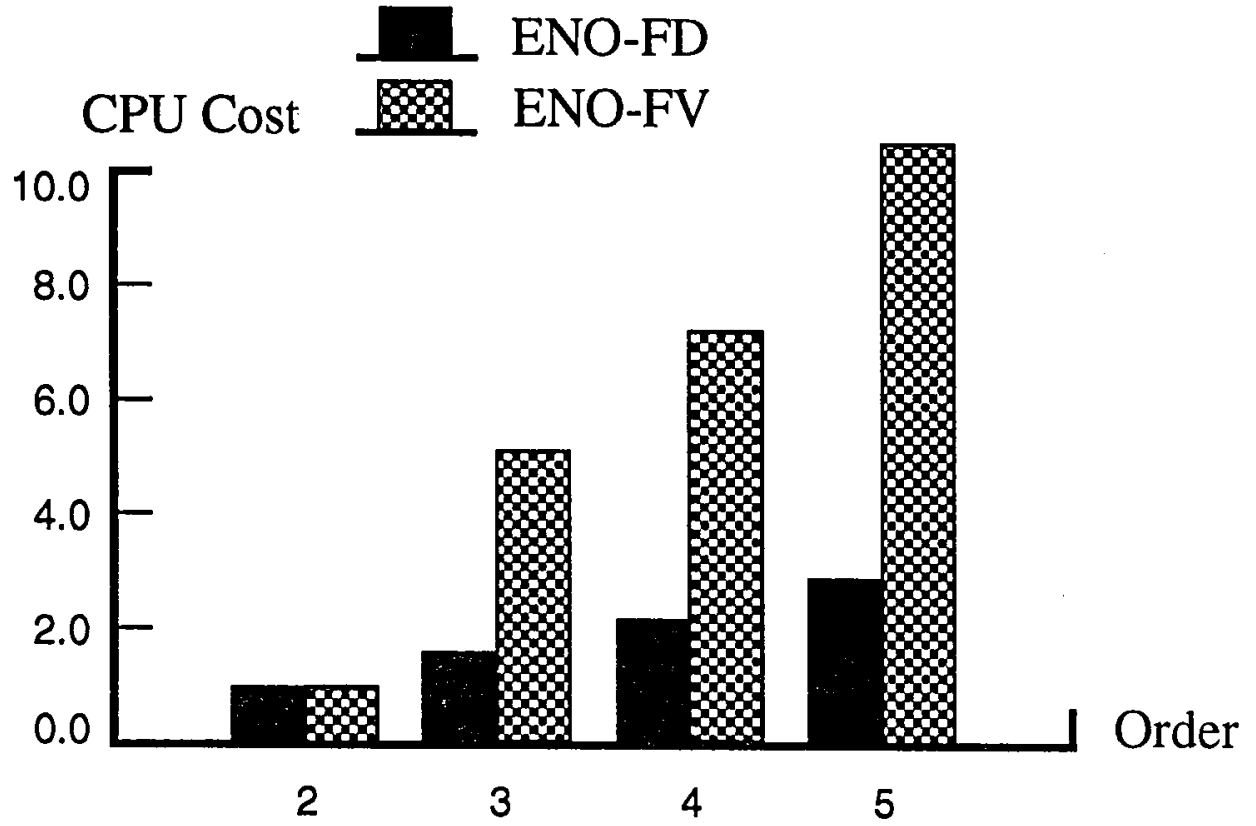

Fig. 16. Relative CPU cost of the two algorithms in three spatial dimensions (estimated). 



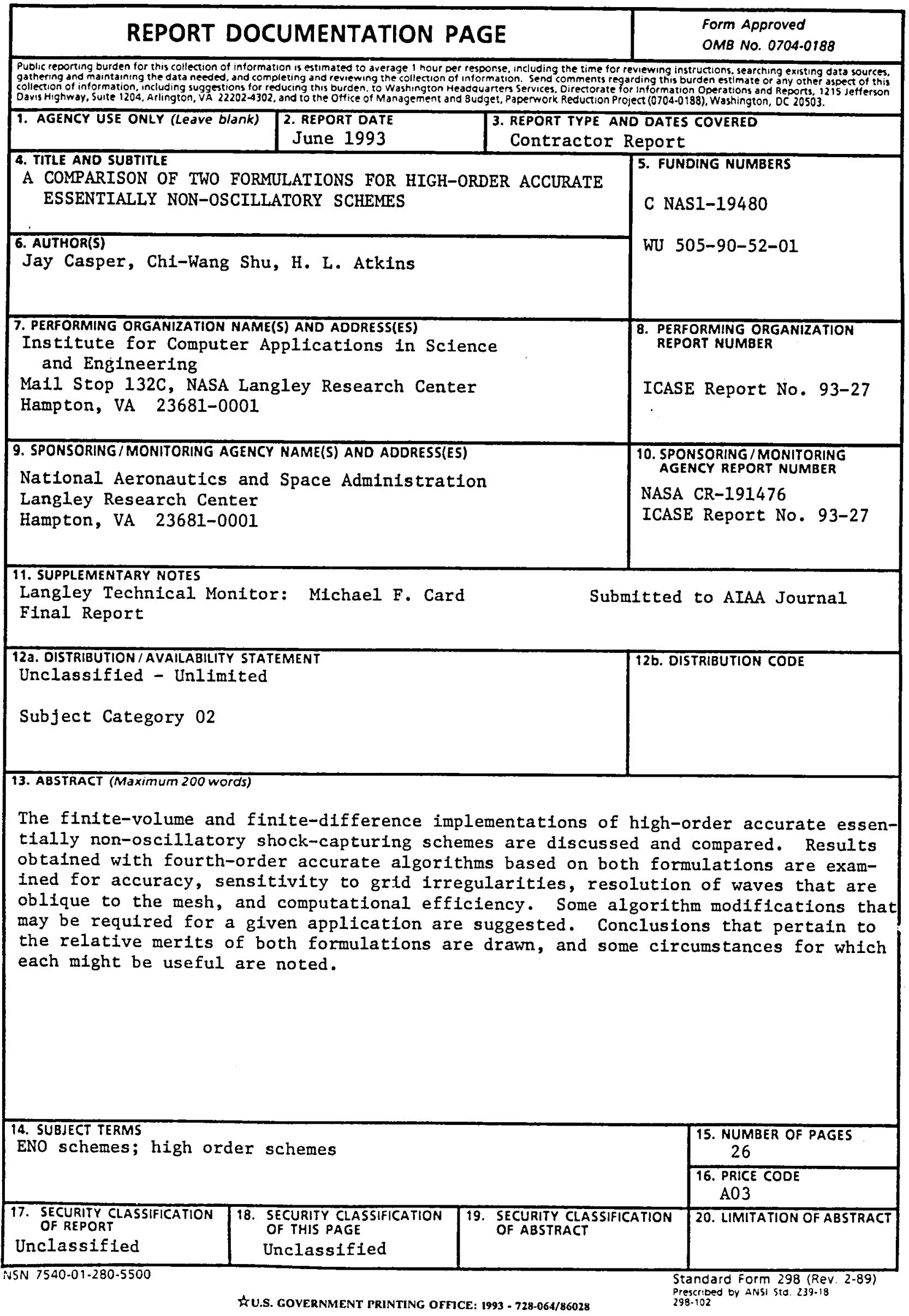




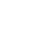


National Aeronautics and Space Administration

\section{Code JTT}

Washington, D.C.

20546-0001

Official Business

Penalty for Private Use, $\$ 300$

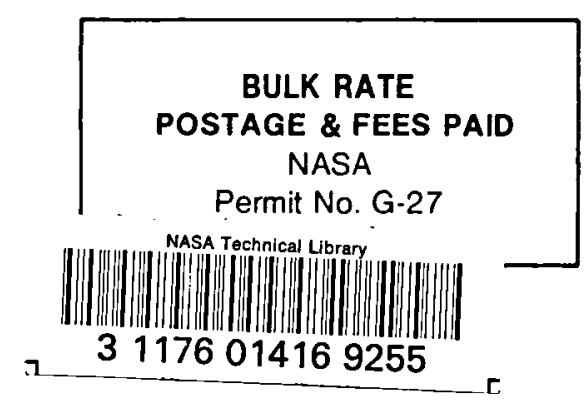

POSTMASTER

If Undeliverable (Section 158 Postal Manual) Do Not Return 